\title{
Economic Dispatch of Power System with Wind Power and Energy Storage Based on Discrete Particle Swarm Optimization
}

\author{
Qiongjie Dai \\ School of Mathematics and Computer Engineering, Ordos Institute of Technology, Ordos, Inner Mongolia, China \\ Email: daiqiongjie06041@163.com
}

\begin{abstract}
In order to solve the economic dispatch problem of power system with wind power and energy storage, the discrete particle swarm optimization (DPSO) algorithm is used to establish the economic dispatch model of power system with wind farm based on chance constrained programming. Discrete particle swarm optimization (DPSO) is used to calculate the minimum generation cost of power system. In the calculation process, the processing constraints of engine group and climbing power are taken into account. In order to verify the effectiveness of the discrete ion algorithm, another algorithm is added to compare. The results show that at the same confidence level, the expected minimum generation cost obtained by the discrete particle swarm optimization (DPSO) algorithm is small, and the time consumed is short. It is a fast search algorithm with good performance.
\end{abstract}

Keywords: Discrete particle swarm optimization, power system, economic dispatch

\section{Introduction}

Nowadays, primary energy is exploited and burned in large quantities all over the world. The problem of energy exhaustion is becoming more and more serious, and it has caused serious environmental pollution. Focusing on the development of renewable energy sources such as wind and solar energy has become the energy strategy of various countries [1,2]. As a renewable energy, wind energy has no pollution and abundant reserves in the world, so it has broad application prospects [3]. The application of wind power technology is more mature than that of other energy sources. In the field of clean energy power generation, wind energy is more efficient than other renewable energy sources such as solar energy [4]. Wind power is the main form of utilization of wind energy. Wind power has many advantages, such as less equipment cost, higher conversion rate and mature power generation technology [5]. Wind power generation has been widely developed all over the world [6]. However, wind energy is a kind of random and discontinuous energy, so it must be combined with a specific energy storage mode to maintain continuous power supply. System is controlled to control wind turbines and generators to ensure the quality of power supply [7]. When using wind energy to generate electricity, since wind energy is random and discontinuous, it is difficult to predict wind power accurately $[8,9]$. When combining wind power generation equipment, harmonics, fluctuation and flicker, three-phase unbalance, voltage sag and other phenomena will occur, which will lead to the unstable operation of the power grid, thus bringing a series of potential safety hazards, which is even more difficult to ensure that the power quality is up to standard.

Particle swarm optimization (PSO) was proposed by Kennedy and Eberhart in 1995. Particle swarm optimization (PSO) arises from the study of bird foraging. The main characteristic of particle swarm optimization is to give each individual a random speed [10] and flow in the whole problem space. Each individual has a memory function. Every individual evolves through cooperation and competition. Particle swarm optimization (PSO) has the advantages of simplicity and easy implementation, as well as deep intelligence. It is not only suitable for scientific research, but also suitable for engineering applications [11]. The problem of economic dispatch of power system with wind power and energy storage has many variables and constraints, and it is a highly non-linear problem, which contains many random variables. The difficulty of solving this problem lies in the treatment of these variables. The key to the problem of economic dispatch of power system is the unbalanced load of the system, which requires a reasonable allocation of the output of each unit in the system to ensure the stable supply of 
power load. At the same time, the stability of system operation, frequency, voltage and function should be guaranteed. The consumption characteristics and operating costs of different units in power system are different, so that different dispatching modes will have economic impact on power system dispatching.

In order to ensure the stability of wind power system operation and maximize the utilization of wind power energy after grid-connected, considering the randomness of wind power, the economic dispatching model based on chance-constrained programming is studied and applied to solve the problem of power system instability. The output of each unit in the power system is allocated and optimized, and the output range and climbing rate of generating units are calculated.

\section{Methodology}

\subsection{Chance-Constrained Programming}

Chance-constrained programming is a key application part of stochastic programming. It has a good application in optimization with stochastic factors at a given confidence level. By using chance constraints, the stochastic problem is transformed into a linear problem. When the constraints contain random variables, decision-making needs to be made in time, but sometimes the realization of the observed random variables is encountered before decision-making is made. Considering this situation, a principle is formulated. The decision-making cannot reach the constraints within a certain level, but the probability of the constraints must be greater than a certain level, which is called confidence level. A common form of opportunity-constrained programming is as follows:

$$
\left\{\begin{array}{l}
\min \bar{f}(x) \\
\text { s.t. } \\
P_{r}\{f(x, \xi) \leq \bar{f}(x)\} \geq \beta \\
P_{r}\left\{g_{i}(x, \xi) \geq 0, j=1,2, \ldots p\right\} \geq \alpha
\end{array}\right.
$$

In the formula, $x$ is an $n$-dimensional decision vector; $\xi$ is a random vector of $\varphi(\xi)$ in a known probability density function; $f(x, \xi)$ is an objective function; $g_{i}(x, \xi)$ is a random constraint function; $P_{r}\{\cdot\}$ is the probability of event establishment in $\{\cdot\} ; \alpha$ and $\beta$ are the pre-given constraint conditions and the confidence level of the objective function, and $\bar{f}$ is the minimum value of the objective function $f(x, \xi)$ when the probability level is at least $\beta$.

\subsection{Mathematical Model for Economic Dispatch of Power System with Wind Farm Based on Chance Constrained Programming}

Objective function: If the operation cost of wind power is not included in the calculation range, the original start-up and shutdown situation will be maintained. Because of the randomness of wind energy, the generation cost of generating units will become a random variable. The objective function is to ensure that the generation cost reaches the lowest expected value.

$$
\min E\left(\sum_{i=1}^{N_{g}}\left(I_{i} f\left(P_{i}^{g}\right)\right)\right)
$$

In the formula, $E[\cdot]$ expresses the mathematical expectation, and the number of generator units added to the optimization is counted as $N_{g} ; I_{i}$ is the operation state of conventional generator unit $i$, the stop is counted as 0 , and $I$ represents the operation; $P_{i}^{g}$ is the active power of conventional generator unit $i ; f\left(P_{i}^{g}\right)$ is the operation cost of conventional generator unit $i$, which is expressed as:

$$
f\left(P_{i}^{g}\right)=a_{i}+b_{i} P_{i}^{g}+c_{i}\left(P_{i}^{g}\right)^{2}
$$

In the formula, $a_{i}, b_{i}$ and $c_{i}$ are the cost coefficients of generator $i$.

Due to the randomness of wind farm output, the constraints contain confidence levels, as follows:

The average power of the system is constrained:

$$
\sum_{i=1}^{N_{g}} C_{i} \cdot P_{i}^{g}+\sum_{k=1}^{N_{w}} P_{k}^{w}=\sum_{j=1}^{N_{t}} P_{j}^{l}
$$


The spinning reserve of the system is constrained:

$$
\begin{gathered}
P_{r}\left\{\sum_{i=1}^{N_{g}} C_{i} \cdot P_{i}^{g \max }-\sum_{i=1}^{N_{g}} C_{i} \cdot P_{i}^{g} \geq R\right\} \geq \beta_{1} \\
R=\eta_{1} P^{L}
\end{gathered}
$$

The climbing ability of the system in minutes is constrained:

$$
P_{r}\left\{\sum_{l=1}^{N_{g}} r_{i}^{u p} P_{i}^{g \max } \geq \sum_{k=1}^{N_{w}} \delta_{k} P_{k}^{w}\right\} \geq \beta_{2}
$$

The ability of transmission lines to transmit active power is constrained:

$$
P_{r}\left\{P_{l}^{l \min } \leq P_{l} \leq P_{l}^{l \max }\right\} \geq \beta_{3}
$$

The output of conventional generators is constrained:

$$
P_{i}^{g \min } \leq P_{i}^{g} \leq P_{i}^{g \max }
$$

In the former formula, the number of wind turbines is counted as $N_{w} ; P_{k}^{w}$ is a random variable, and the active power output of wind turbines is counted as k; The number of load buses is counted as $N_{t} ; P_{j}^{l}$ is the load demand of bus $j ; P_{r}\{\cdot\}$ is the probability of event establishment in $\{\cdot\} ; \beta_{1}, \beta_{2}, \beta_{3}$ are a certain confidence level; $P_{i}^{g \max }$ and $P_{i}^{g \min }$ are the maximum power and minimum power of generator i; The rotating spare active power of the system is recorded as $R ; \eta_{1}$ is the reserve rate of the system; $P^{L}$ is the total load of the system; $r_{i}^{u p}$ is the highest climbing rate that can be achieved by generating unit $i$ in the case of output active power in minute stage; $\delta_{k}$ is the maximum output that wind power unit can possibly achieve in range; $P_{l}^{l \max }$ and $P_{l}^{l \min }$ are the upper and lower limits of transmission active power for line $l$.

When predicting the output of wind farm, the Weibull function can be regarded as the treatment of long time, and the wind force can be approximated as the normal distribution in the case of short time. The output of wind turbine can be calculated by formula 10 .

$$
P=\left\{\begin{array}{lc}
0 & \mu \leq \mu_{c i}, \mu \geq \mu_{\infty} \\
A+B \mu+C^{2} \mu^{2} & \mu_{c i} \leq \mu \leq \mu_{r} \\
P_{r} & \mu_{r} \leq \mu \leq \mu_{\infty}
\end{array}\right.
$$

In the formula, the parameters of fan power characteristic curve are recorded as $A, B$ and $C$. Wind will vary slightly in different regions. $C$ is sometimes taken as zero in approximate calculation, that is to say, wind speed and output are linear. $\mu_{c i}, \mu_{r}, \mu_{\infty}$ and $P_{r}$ are respectively the starting wind speed, rated wind speed, resection wind speed and rated power of the fan. In the wind farm, when the wind passes through the generator set, the wind speed will decrease to a certain extent, and the smaller the distance between the wind turbine sets is, the greater the impact is. This interaction is called wake effect. The energy loss caused by wake effect may be an important reason why the energy conversion rate of wind farms cannot be improved, and it also has a serious impact on the economy of wind farms.

\subsection{Particle Swarm Optimization}

Random simulation technology provides an effective way to verify the constraints in the form of probability, considering the opportunity constraints:

$$
P_{r}\left\{g_{j}(x, \xi) \leq 0, j=1,2 \ldots, k\right\} \geq \alpha
$$

In the formula, $\xi$ is a random variable with probability density function of $\varphi(\xi)$. For a certain decision variable $x, N_{1}=0$. Then, $N$ random variables are obtained. If the obtained random variables $\xi$ and decision variables $x$ are substitute into equation 11 , the inequality $g_{i}(x, \xi) \leqslant 0$, and $j=1,2, \ldots$ If $k$ holds, $N_{1}$ plus 1 . When $N$ is large enough, according to the law of large numbers, formula 11 holds, if and only if $N_{1} / N \geqslant \alpha$ holds. If $N_{1} / N \geqslant \alpha$ is not valid, it means that the particles generated by PSO do not conform to the probability level in formula 11. Therefore, such particles should be discarded and new particles should be obtained for calculation until all the particles obtained satisfy $N_{1} / N \geqslant \alpha$.

\subsection{Specific Solution Process}

According to the model, the process of using discrete particle swarm optimization (DPSO) is as follows. 
Firstly, the number of generating units, installed capacity, operation parameters of wind turbines and random wind speed parameters of wind farms are recorded.

Secondly, the basic values of conventional generating units in the system are input, such as economic values, output limitations, reserve requirements, etc.

Thirdly, the confidence level of the constraints with random variables is set. The parameters of the discrete particle swarm optimization algorithm are input: termination conditions (usually iterations), population size pop-size;

Fourthly, the random number generator is used to generate the adjustable output of conventional generators and the random wind speed with normal distribution, and the output of wind farm is calculated to check whether the particles can reach the confidence level of the constraint conditions and form pop-size feasible initial particle swarm positions and velocities.

Fifthly, the fitness of each particle is calculated.

Sixthly, the fitness of each particle is compared with the individual extreme value. If it is better, the current individual extreme value $P_{\text {best } i}$ is updated.

Seventhly, for each particle, its fitness is compared with the global extremum. If it is better, the current global extremum $G_{\text {best }}$ is updated.

Eighthly, according to formula 12 and 13, the position and velocity of each particle are updated, and whether the updated particle meets the requirement of confidence level constraint is checked. If not, the particle is regenerated and its position updated until the constraints are met. If the number of repeated updates exceeds the specified number of times (such as 100 times), the original feasible particle's $P_{\text {best } i}$ is used instead.

$$
\begin{gathered}
v_{i}^{n+1}=w \times v_{i}^{n}+c_{1} \times r_{1} \times\left(P_{\text {best }}^{n}-x_{i}^{n}\right)+c_{2} \times r_{2} \times\left(G_{\text {best }}-x_{i}^{n}\right) \\
x_{i}^{n+1}=x_{i}^{n}+v_{i}^{n+1} \\
w=1.05-n * \frac{1.05-0.4}{c}
\end{gathered}
$$

By changing w dynamically, the computational speed of discrete particle swarm optimization (DPSO) can be improved. The range of $\mathrm{w}$ values can directly affect the accuracy and speed of convergence. Through many experiments, it is found that when the range of $\mathrm{w}$ values is $[1.05,0.4]$, it can not only guarantee faster convergence speed, but also prevent premature convergence of population. In the formula, $\mathrm{n}$ is the current number of cycles. $c_{1}=c_{2}=2 . c$ is the total number of cycles specified. $r_{1}$ and $r_{2}$ are random numbers between $(0,1) . x_{i}$, and $v_{i}$ are the position and velocity of the particle in the $i$-th dimension.

Ninthly, steps $(5) \sim(8)$ are repeated until the termination conditions are met.

Tenthly, the best particle is given as the optimal solution. The flow chart is shown in figure 1 .

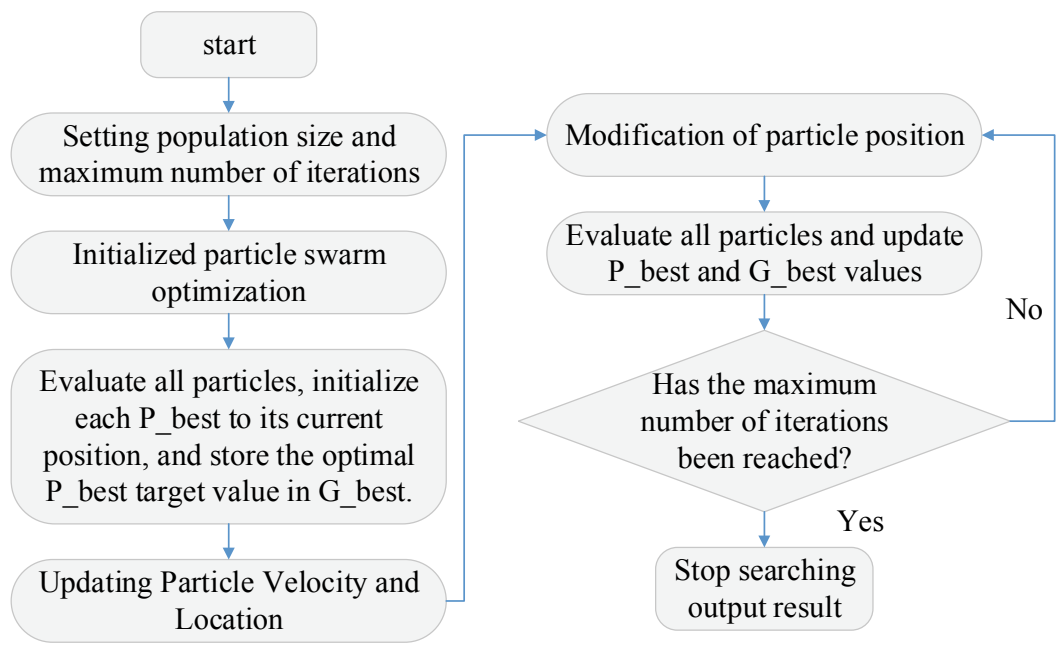

Figure 1. Particle swarm optimization for economic scheduling 
Taking the standard test system of IEEE-30 bus as the research object, if the wind power is connected to the system at 15 nodes, taking the $600 \mathrm{~kW}$ variable-pitch wind turbines as an example, there are 25 wind turbines, whose cut-in wind speed, cut-out wind speed and rated wind speed are 3,25 and $14 \mathrm{~m} / \mathrm{s}$, respectively. Assuming that the 12-node and 14-node generators must be turned on, and the generating power is fixed with a fixed value, the generator set on one node is fixed, and the generator set on the other nodes can be adjusted under the condition of output. In order to verify the effectiveness of the proposed algorithm, another algorithm is added to study, and the results of the two algorithms are collected for comparison and verification. The genetic algorithm (algorithm 1) based on stochastic simulation and the discrete particle swarm optimization (algorithm 2) proposed in this study are commonly used to solve chance-constrained programming.

\section{Results and Discussion}

\subsection{Generator Component Cost}

In the standard test system of IEEE-30 nodes, wind power is connected to the system at 15 nodes. Under the standard of $600 \mathrm{~kW}$ variable-pitch wind turbine with single unit capacity, the standby level of rotation is based on $15 \%$ of the system load, and the maximum variation of wind turbine output is considered at $30 \%$. Among them, $\mu=7.5$ and $\delta=2$. The relationship between wind turbine output and wind speed is shown in figure $2 \mathrm{~A}$, and the relevant parameters of the generator are shown in figure $2 \mathrm{~B}-\mathrm{D}$.
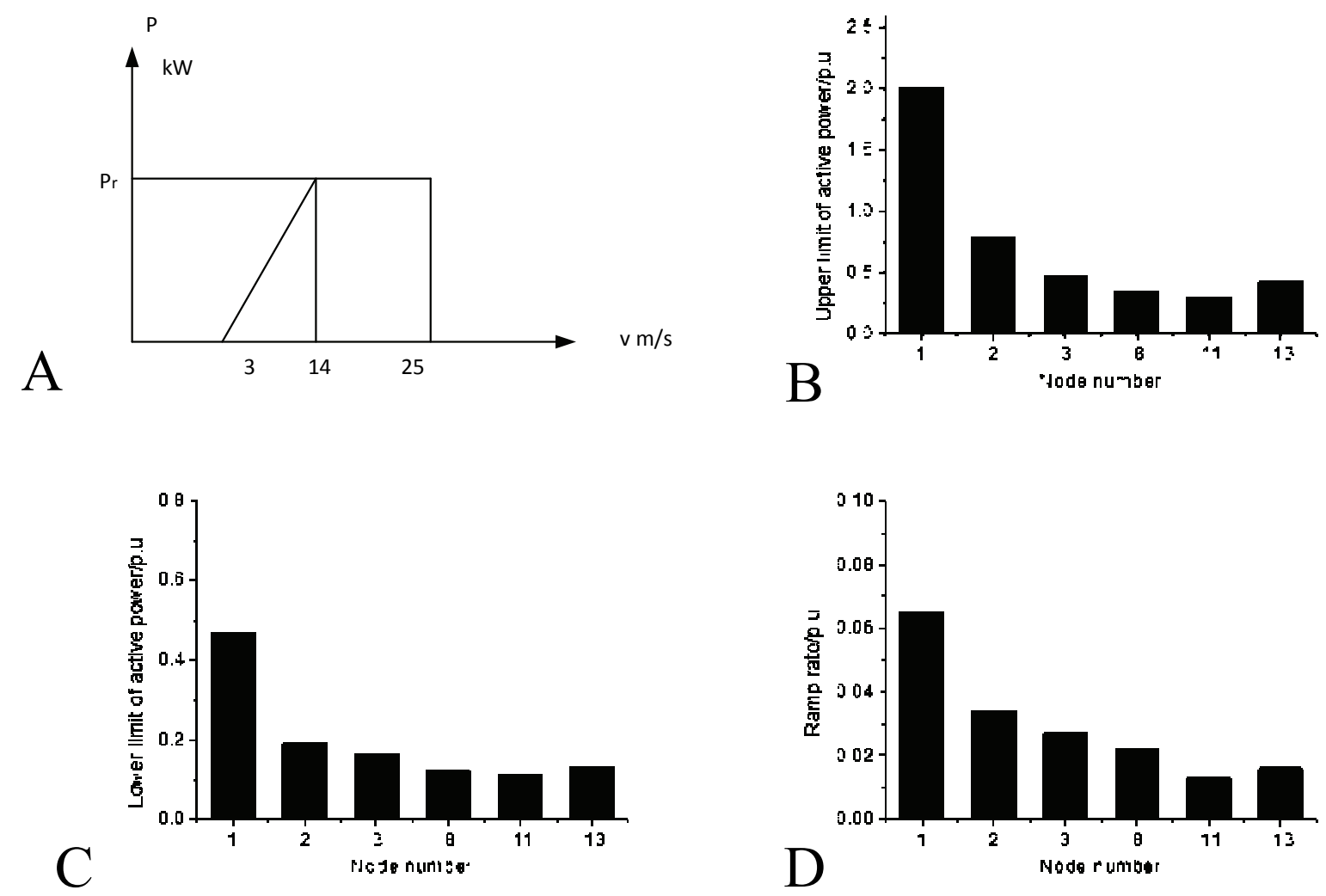

Figure 2. Generator parameters (A: the relationship between generator output and wind speed B: upper limit of active power for each node of the generator set C: lower limit of active power for each node of the generator set D: climbing speed of each node of the generator set) 


\subsection{Computation Results of Different Algorithms}

Under the same confidence level, the results show that the minimum generation cost calculated by the discrete particle swarm optimization algorithm is less than the expected minimum generation cost obtained by the genetic algorithm based on stochastic simulation, and the calculation time of the discrete particle swarm optimization algorithm is shorter. When the confidence level is lowered and the calculation is made again, if it is changed from 0.97 to 0.94 , the generation cost will be reduced slightly, which indicates that the minimum expected generation cost will also be reduced when the reliability requirement is reduced. The main reason is that $\beta_{1}$ and $\beta_{2}$ are almost $100 \%$ satisfied in the process of calculation. Only the power flow on line 1-2 will become more and more restrictive, that is, $\beta_{3}$ cannot always guarantee the probability level of 0.97 , so changing the confidence level, $\beta_{3}$ will change the size of the expected generation cost as the main influencing factor. The size of the system is smaller, and the change of the expected minimum generation cost will be less, as shown in figure 3 .
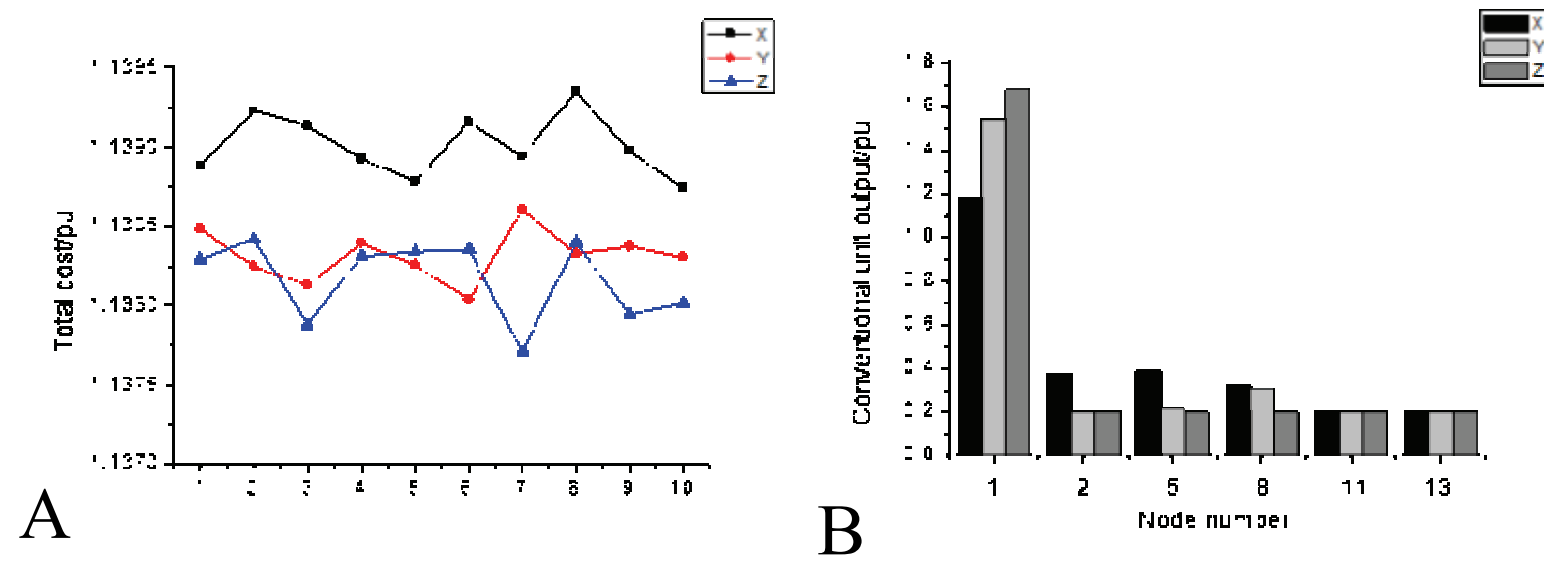

Figure 3. Comparison of two algorithms (A: Comparing the total generation cost of regular units B: Optimal solution for ignoring network loss) (In the figure, $\mathrm{X}$ shows the expected minimum generation cost when the confidence levels of algorithm $1, \beta_{1}, \beta_{2}$ and $\beta_{3}$ are $0.97 ; \mathrm{Y}$ is the expected minimum generation cost when the confidence levels of algorithm $2, \beta_{1}, \beta_{2}$ and $\beta_{3}$ are $0.97 ; \mathrm{Z}$ is the expected minimum generation cost when the confidence levels of algorithm $1, \beta_{1}, \beta_{2}$ and $\beta_{3}$ are $\left.0.94 ;\right)$

\section{Conclusion}

In this study, according to the randomness and unpredictability of wind power, discrete particle swarm optimization (DPSO) is used to solve the economic dispatch of power system with wind power and energy storage. The results show that after the economic dispatch model of chance-constrained programming is constructed and the output of each unit is allocated according to the algorithm, the constraints of a certain probability can be satisfied under the condition of allowing the wind speed to change randomly, and the expected cost can be minimized. Discrete particle swarm optimization (DPSO) is used to calculate the minimum generation cost of power system, which is less than the expected minimum generation cost based on stochastic simulation genetic algorithm, and the calculation time of DPSO is shorter. When the confidence level is lowered, the generation cost can be reduced slightly, which indicates when the requirement of reliability is reduced, the minimum expected generation cost will also be reduced. It also shows that particle swarm optimization is an excellent algorithm in solving the economic dispatch problem which takes a long time.

Acknowledgements. This research was funded by Scientific Research Project of Universities in Inner Mongolia (Grant number: NJZC17415) and Research Project of Ordos Institute of Technology (Grant number: KYYB2017013). 


\section{References}

1. Gong $\mathrm{M}$, Wu Y, Cai Q, et al. Discrete particle swarm optimization for high-order graph matching [J]. Information Sciences, 2016, 328, pp. 158-171.

2. Guo F, Wen C, Mao J, et al. Distributed economic dispatch for smart grids with random wind power [J]. IEEE Transactions on Smart Grid, 2015, 7(3), pp. 1572-1583.

3. Li G, Zhang R, Jiang T, et al. Security-constrained bi-level economic dispatch model for integrated natural gas and electricity systems considering wind power and power-to-gas process [J]. Applied energy, 2017, 194, pp. 696704.

4. Jiang $\mathrm{Y}, \mathrm{Xu}$ J, Sun $\mathrm{Y}$, et al. Day-ahead stochastic economic dispatch of wind integrated power system considering demand response of residential hybrid energy system [J]. Applied energy, 2017, 190, pp. 1126-1137.

5. Peng C, Xie P, Pan L, et al. Flexible robust optimization dispatch for hybrid wind/photovoltaic/hydro/thermal power system [J]. IEEE Transactions on Smart Grid, 2015, 7(2), pp. 751-762.

6. Qu B Y, Liang J J, Zhu Y S, et al. Economic emission dispatch problems with stochastic wind power using summation based multi-objective evolutionary algorithm [J]. Information Sciences, 2016, 351, pp. 48-66.

7. Qu B Y, Liang J J, Zhu Y S, et al. Economic emission dispatch problems with stochastic wind power using summation based multi-objective evolutionary algorithm [J]. Information Sciences, 2016, 351, pp. 48-66.

8. Bizuayehu A W, de la Nieta A A S, Contreras J, et al. Impacts of stochastic wind power and storage participation on economic dispatch in distribution systems [J]. IEEE Transactions on Sustainable Energy, 2016, 7(3), pp. 1336-1345.

9. Wang X, Jiang C, Li B. Active robust optimization for wind integrated power system economic dispatch considering hourly demand response [J]. Renewable energy, 2016, 100(97), pp. 798-808.

10. Cai Q, Gong M, Ma L, et al. Greedy discrete particle swarm optimization for large-scale social network clustering [J]. Information Sciences, 2015, 316, pp. 503-516.

11. Beheshti Z, Shamsuddin S M, Hasan S. Memetic binary particle swarm optimization for discrete optimization problems [J]. Information Sciences, 2015, 299, pp. 58-84. 